\title{
Strength of patient cohorts and biobanks for cardiomyopathy research
}

\author{
R. A. de Boer · L. L. A. M. Nijenkamp · H. H. W. Sillé · T. R. Eijgenraam • R. Parbhudayal • B. van Driel • R. Huurman • \\ M. Michels - J. Pei · M. Harakalova · F. H. M. van Lint · M. Jansen - A. F. Baas · F. W. Asselbergs · J. P. van Tintelen · \\ B. J. J. M. Brundel · L. M. Dorsch · M. Schuldt · D. W. D. Kuster · J. van der Velden for DOSIS consortium
}

(C) The Author(s) 2020

\begin{abstract}
In 2011 the Netherlands Heart Foundation allocated funding (CVON, Cardiovasculair Onderzoek Nederland) to stimulate collaboration between clinical and preclinical researchers on specific areas of research. One of those areas involves genetic heart diseases, which are frequently caused by pathogenic variants in genes that encode sarcomere proteins. In 2014, the DOSIS (Determinants of susceptibility in inherited cardiomyopathy: towards novel therapeutic approaches) consortium was initiated, focusing their research on secondary disease hits involved in the onset and progression of cardiomyopathies. Here we highlight several recent observations from our consortium and collaborators which may ultimately be relevant for clinical practice.
\end{abstract}

\section{R. A. de Boer · H. H. W. Silljé · T. R. Eijgenraam} Department of Cardiology, University Medical Center Groningen, University of Groningen, Groningen, The Netherlands

\section{R. Huurman · M. Michels}

Department of Cardiology, University Medical Center Rotterdam, Erasmus MC, Rotterdam, The Netherlands

J. Pei · M. Harakalova · F. W. Asselbergs

Department of Cardiology, Division Heart \& Lungs, University Medical Centre Utrecht, Utrecht University, Utrecht, The Netherlands

F. H. M. van Lint · M. Jansen · A. F. Baas · J. P. van Tintelen Department of Genetics, University Medical Centre Utrecht, Utrecht University, Utrecht, The Netherlands

L. L. A. M. Nijenkamp · R. Parbhudayal · B. van Driel · B. J. J. M. Brundel · L. M. Dorsch · M. Schuldt .

D. W. D. Kuster · J. van der Velden $(\triangle)$

Department of Physiology, Amsterdam Cardiovascular Sciences, Amsterdam UMC, Vrije Universiteit, Amsterdam, The Netherlands

j.vandervelden1@amsterdamumc.nl
Keywords Cardiomyopathies - Gene variants . Patient cohorts

\section{Introduction}

Inherited cardiomyopathies, caused by pathogenic variants in genes encoding proteins that regulate cardiomyocyte contractility, are a major cause of morbidity and mortality. In $50-60 \%$ of familial hypertrophic cardiomyopathy (HCM) and $30-40 \%$ of dilated cardiomyopathy (DCM), a pathogenic gene variant can be identified. The most common genes that are affected in HCM are MYH7, MYBPC3 and TNNT2, which encode the thick filament proteins myosin heavy chain, cardiac myosin-binding protein-C (cMyBP$\mathrm{C}$ ), and the thin filament protein troponin T. In DCM the titin (TTN) gene, which encodes the giant myofilament protein titin, is the most frequently affected gene ( $15-20 \%$ of all gene variants) [1]; in particular gene variants that lead to $T T N$ truncation have been shown to be pathogenic [2]. In addition, in the Netherlands, a founder mutation in the PLN gene, which encodes the calcium-handling protein phospholamban, was

Dutch contribution to the field

- DOSIS represents a national research consortium on cardiomyopathies.

- DOSIS researchers have shown more severe diastolic dysfunction in female than male HCM patients at the time of surgery.

- Indexation for body size is needed to set the diagnostic threshold for left ventricular thickening in HCM.

- Cell-to-cell variability is present in the hearts of patients with a Dutch founder mutation. 


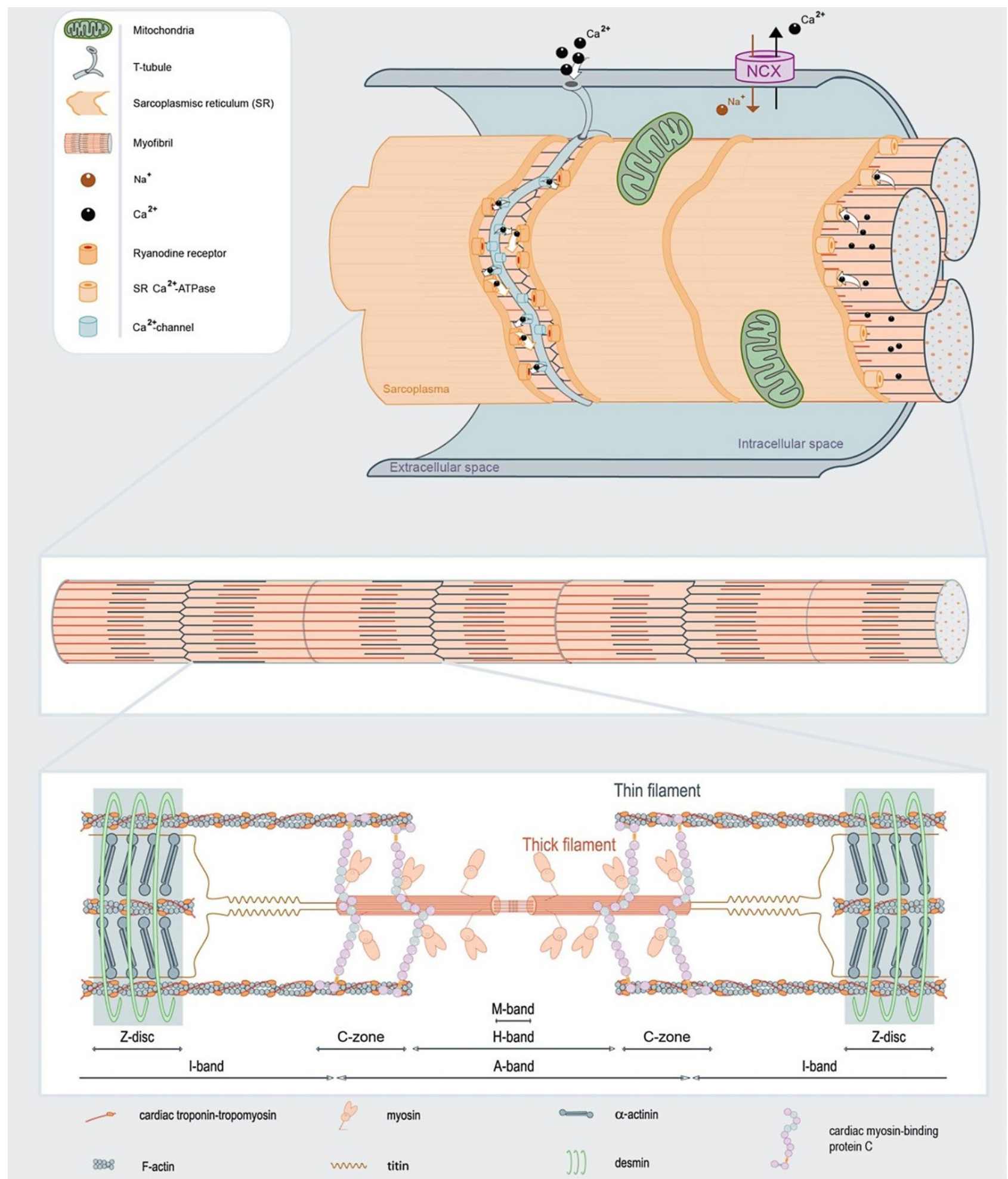

Fig. 1 Activation of a cardiomyocyte triggers calcium entry and release of calcium from the sarcomplasmic reticulum $(S R)$, which results in contraction of the cardiac myofibrils. To relax the cardiomyocyte, calcium is pumped back into the SR, and out of the cell via the sodium-calcium exchanger (NCX). Myofibrils consist of sarcomeres composed of the thin actin and thick myosin filament, and the third filament titin. Sarcomeres consist of sub-regions (depicted by the different bands), which underlie the striated pattern of cardiac muscle. Gene variants that cause cardiomyopathies are frequently found in myosin heavy chain, troponin T, cardiac myosin-binding protein-C (located in the Z-zone) and titin. (Figure is adapted from Sequeira at al. [34]) 
Fig. 2 Disease modifiers in inherited cardiomyopathies (PQC protein quality control)

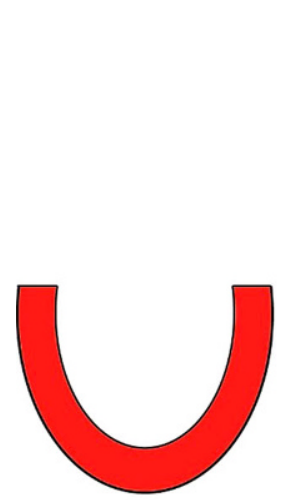

Asymptomatic carrier
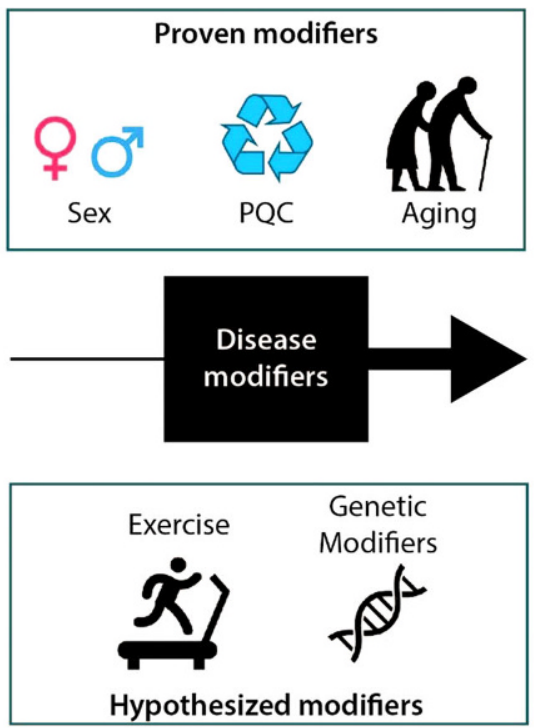

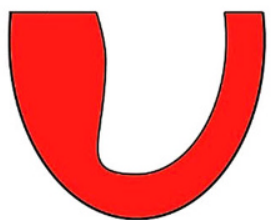

Hypertrophic cardiomyopathy

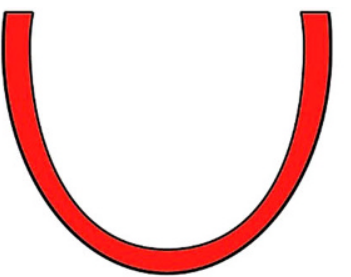

Dilated cardiomyopathy identified in 2012 as a now well-known cause of DCM and arrhythmogenic cardiomyopathy [3, 4]. Fig. 1 depicts a cardiomyocyte to illustrate the affected proteins involved in cardiomyopathies. Upon activation of a cardiomyocyte, calcium enters the cell via the L-type calcium channel, which subsequently releases calcium from the intracellular calcium store, the sarcoplasmic reticulum. Calcium binds to the troponin complex, which induces a conformational change of troponin-tropomyosin, and thereby releases binding sites for myosin heads on the thin actin filament. Binding of myosin to actin (so-called cross-bridge) results in force development. The kinetics of crossbridge cycling is regulated by cMyBP-C, and the giant protein titin, encoded by TTN and linked with DCM, underlies passive stiffness of sarcomeres [5].

In past years the number of genes in genetic diagnostic panels has increased with the hope to identify a disease-causing variant in a larger group of patients and their family members. However, recent studies conclude that the additional benefit of screening large numbers of genes is disappointingly low and of marginal clinical utility [6, 7]. Numerous new TTN gene variants have been identified, mainly because of its large size. A study in three European cardiogenic centres showed that missense and non-frameshifting insertions/deletions variants are most likely benign, as reference populations showed comparable frequencies of these rare TTN variants [8]. The current panels thus rather increase the number of variants with unknown significance, which are likely benign, though they may have a modifier role in disease. Cardiomyopathy patients with a suspected genetic aetiology should be referred for genetic screening. For HCM this includes patients with asymmetric left ventricular hypertrophy, not explained by abnormal loading conditions, with or without a clear family history. For DCM this includes patients with nonischaemic DCM, not fully explained by other aetio- logical factors. Young age of onset and familial occurrence are important parameters that hint towards a genetic aetiology. However, late onset in seemingly sporadic cases does not exclude a genetic origin due to the reduced penetrance and variable disease expression. All HCM and DCM patients in whom genetic screening was performed can be added to the biobanks, including their relatives (asymptomatic mutation carriers).

Since cardiomyopathies continue to constitute one of the most common causes of sudden cardiac death in the young and still represent major causes for cardiac transplantation, adequate identification of additional disease triggers and understanding the pathomechanisms is of utmost importance. The clinical approach is furthermore complicated since inherited cardiomyopathies are clinically heterogeneous: age-dependent penetrance and disease-severity differ greatly between patients with the identical genetic variant. The mechanisms that underlie the variation in disease expression are still largely unknown. By combining cellular, genetic and clinical data from well-phenotyped national patient cohorts, DOSIS strived to define disease factors (i.e. secondary hits) that in addition to the pathogenic gene variant cause and aggravate cardiac disease in cardiomyopathy patients (Fig. 2; Tab. 1). Several recent observations are highlighted below.

\section{Indexation for body size to set the diagnostic threshold for left ventricular thickening}

Using a large collection of myectomy samples from patients with obstructive HCM, we have shown that there is a sex-specific difference in diastolic function at the time of myectomy in HCM patients carrying pathogenic variants in MYH7 and MYBPC3 [9]. Women showed more diastolic dysfunction evident from significantly higher E/e' ratios, impaired left 
Table 1 Cohorts of DOSIS

\begin{tabular}{|c|c|c|c|c|}
\hline Cohort & Participating centres & Population & Biobank collection & Aim \\
\hline $\begin{array}{l}\text { Erasmus HCM observa- } \\
\text { tional cohort }\end{array}$ & Erasmus MC & $\begin{array}{l}\text { HCM patients and } \\
\text { gene variant carriers }\end{array}$ & DNA & $\begin{array}{l}\text { Identify predictive clinical markers for major cardiac } \\
\text { events }\end{array}$ \\
\hline $\begin{array}{l}\text { BIO FOr CARe observa- } \\
\text { tional cohort }\end{array}$ & $\begin{array}{l}\text { UMC Utrecht, UMC Gronin- } \\
\text { gen, Amsterdam UMC, } \\
\text { Erasmus MC }\end{array}$ & $\begin{array}{l}\text { All sarcomere gene } \\
\text { variants }\end{array}$ & $\begin{array}{l}\text { DNA, RNA (from } \\
\text { blood), plasma and } \\
\text { serum }\end{array}$ & $\begin{array}{l}\text { Identify predictive biomarkers for major cardiac } \\
\text { events }\end{array}$ \\
\hline Telephone interviews & $\begin{array}{l}\text { UMC Utrecht, UMC Gronin- } \\
\text { gen, Amsterdam UMC }\end{array}$ & $\begin{array}{l}\text { MYBPC3 founder } \\
\text { gene } \\
\text { variant carriers }\end{array}$ & n.a. & $\begin{array}{l}\text { Determine predictive value of environmental factors } \\
\text { (especially exercise) for major cardiac events }\end{array}$ \\
\hline $\begin{array}{l}\text { ENERGY randomised } \\
\text { placebo-controlled trial }\end{array}$ & $\begin{array}{l}\text { Amsterdam UMC, Erasmus } \\
\text { MC }\end{array}$ & $\begin{array}{l}\text { Preclinical } M Y H 7 \\
\text { gene variant carriers }\end{array}$ & Serum & $\begin{array}{l}\text { Determine effects of trimetazidine on improving my- } \\
\text { ocardial energy efficiency in the pre-clinical disease } \\
\text { stage }\end{array}$ \\
\hline Myectomy cohort & Erasmus MC, UMC Utrecht & $\begin{array}{l}\text { HCM patients un- } \\
\text { dergoing septal } \\
\text { myectomy }\end{array}$ & DNA, cardiac tissue & Collect myocardial tissue for use in etiological studies \\
\hline
\end{tabular}

ventricular filling patterns, and higher tricuspid regurgitation velocities. Of the female patients, $50 \%$ showed grade III diastolic dysfunction, while the majority of male patients (56\%) had only mild (grade I) diastolic dysfunction. Correction of maximal septal thickness and left atrial diameter for body surface area (BSA) resulted in significantly higher values in female compared with male patients. Histological and protein analyses revealed more advanced remodelling of the heart in female compared with male HCM patients evident from higher levels of fibrosis and activation of the cardiac foetal gene program, which is characteristic of heart failure. In addition to genetic screening, the current diagnostic criterion of hypertrophy is a maximal left ventricular wall thickness of $\geq 15 \mathrm{~mm}$, or $\geq 13 \mathrm{~mm}$ in first-degree relatives of HCM patients. As the hearts of women, and in general relatively small persons, are smaller than the hearts of men, this threshold for the diagnosis of HCM should probably be corrected for body size [10]. The current diagnostic threshold, which does not take into account body size, may likely explain the male predominance in HCM patient cohorts, simply because males in general have larger hearts. A recent study in a Dutch cohort of 199 genotype-positive subjects, family members of HCM patients who were referred for cardiac screening between 1995 and 2018, indexation of wall thickness by BSA decreased the number of HCM diagnoses [11]. Moreover, predictive accuracy for HCM-related events (mortality, cardiac transplantation, implantable cardioverterdefibrillator implantation and septal reduction therapy) improved significantly after indexation by BSA. These studies indicate that correcting left ventricular thickness for body size should be considered for the diagnosis of HCM and longitudinal follow-up studies in larger cohorts of preclinical genotype-positive individuals are needed to confirm this.

\section{Altered metabolism as key driver of disease in cardiomyopathies}

Several studies suggest an important role for secondary disease modifiers such as additional epigenetic and genetic variations and environmental disease triggers. Compelling data have accumulated that obesity is an overarching risk factor, also for age-of-onset and severity of cardiomyopathies. Proof that obesity contributes to disease onset and severity comes from cohort studies. The international HCM Share registry showed that patients with a high body mass index have a significantly increased risk of heart failure, more advanced left ventricular outflow tract obstruction and more arrhythmias (i.e. HCM-related outcomes) [12]. Moreover, a prospective study in adolescent men demonstrated that even mildly elevated body weight in late adolescence significantly increased the risk to develop dilated cardiomyopathy in adulthood [13]. At the heart level, a recent proteomics study in human HCM tissue samples showed reduced levels of energy metabolism proteins [14]. This observation is in line with studies in human HCM showing energy deficiency of the heart [15, 16]. Energy deficiency has been proposed as the primary variant-induced pathomechanism of HCM [17], which is supported by studies showing reduced cardiac efficiency in preclinical asymptomatic carriers of sarcomere gene variants in the absence of cardiac hypertrophy $[16,18]$. Accordingly, DCM caused by TTN gene variants has been linked with mitochondrial dysfunction and metabolic perturbations as cause of disease progression [19]. Overall, these studies indicate that timely disease stage-specific treatment of metabolic perturbations may slow down disease progression in cardiomyopathy patients [20]. An observational cohort to determine the predictive value of metabolic biomarkers (BIO FOr CARe: identification of biomarkers for development and progression of HCM in carriers of the Dutch MYBPC3 founder carriers) and a clinical trial using metabolic drug therapy aimed to improve energetics of the heart at preclinical 
Fig. 3 By combining cellular, genetic and clinical data from well-phenotyped national patient cohorts, DOSIS strives to define disease factors that in addition to the pathogenic gene variant cause and aggravate cardiac disease in cardiomyopathy patients

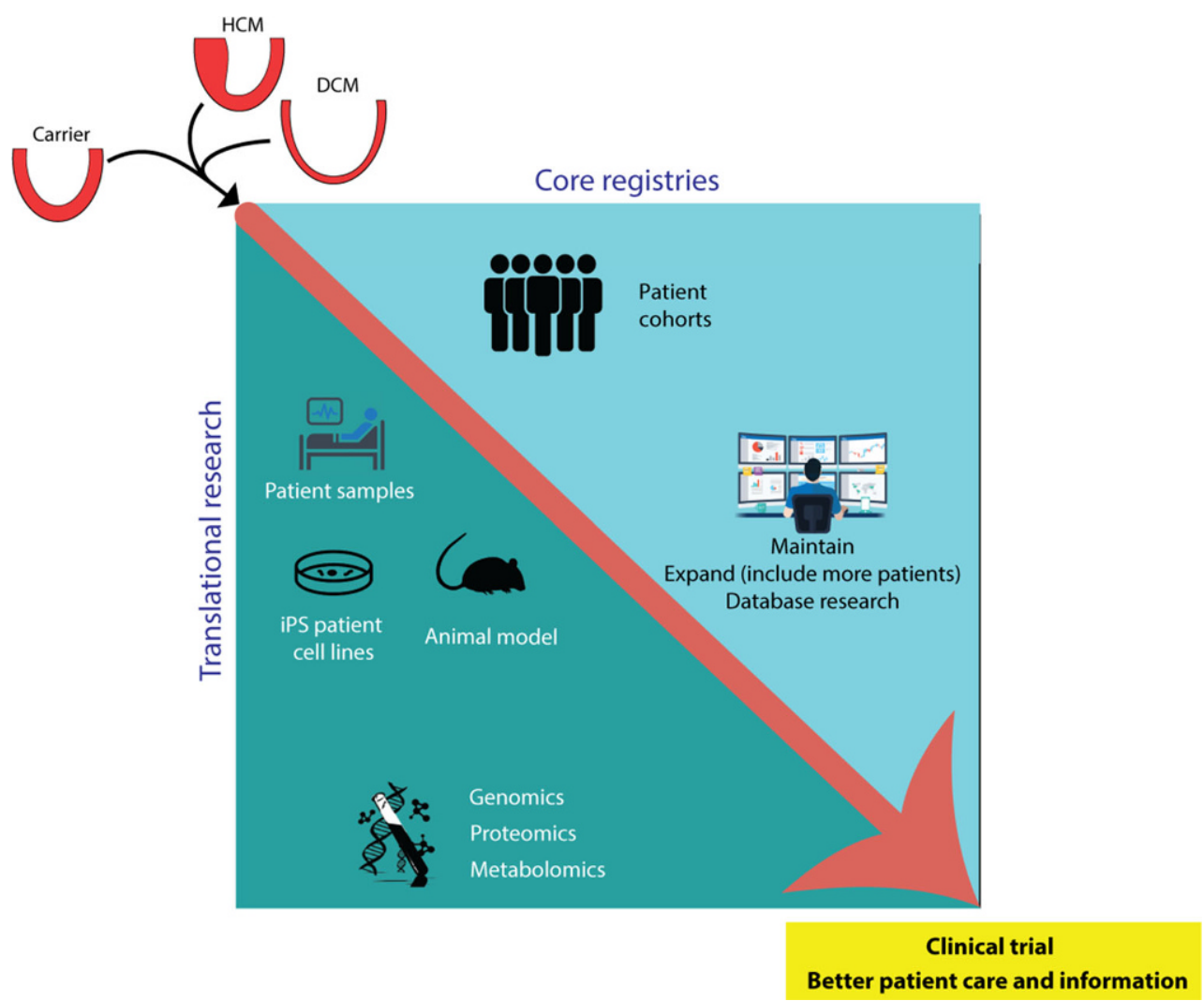

stage in HCM gene variant carriers are currently being performed by several DOSIS principal investigators (ENERGY trial) [21].

\section{Altered protein quality control as disease modifier in cardiomyopathy}

An age-related decline in protein quality control (PQC) has been proposed as a contributor to disease progression in cardiomyopathy. As sarcomere proteins are the most abundant proteins in the heart, maintenance of sarcomere structure and function depends on PQC mechanisms. Pathogenic gene variants result in poison polypeptides or reduced protein levels (haploinsufficiency) and may trigger PQC and/or stress cellular protein homeostasis. DCM patients with truncating $T T N$ variants show a relatively mild disease course, though with significant excess mortality in elderly patients. The latter may be explained by an age-related deterioration of the PQC mechanisms. As life expectancy increases, $T T N$-associated morbidity and mortality will likely become more prevalent [22]. Also in PLN-associated cardiomyopathy, protein aggregation and activation of PQC pathways has been observed in end-stage disease [23].

Terminally misfolded and aggregation-prone proteins are cleared by the two degradation systems, the ubiquitin-proteasome system and autophagy [24]. Furthermore, pathways of PQC are strongly linked to cell architecture, such as the microtubules net- work. DOSIS studies in a large set of cardiac tissues from a well-characterised HCM patient group showed altered PQC with several specific changes in gene-variant positive patients (genotype-positive) compared with genotype-negative patients and nonfailing controls [25]. Heat shock proteins (HSP) involved in protein stabilisation (HSPB1) and refolding (HSPD1, HSPA2) were increased in genotype-positive HCM compared with controls. In addition, tubulin and acetylated-tubulin levels were significantly higher in HCM compared with controls, especially in HCM with truncating variants in $M Y B P C 3$, which cause protein haploinsufficiency. cMyBP-C protein levels were inversely correlated with $\alpha$-tubulin levels suggesting a compensatory tubulin response to maintain cardiomyocyte structure, though this may be at the expense of cardiac function. Our study indicates that proliferation of the microtubular network represents a novel pathomechanism in cMyBP-C haploinsufficiency-mediated HCM. Recent studies in human heart failure identified a central role for detyrosinated microtubules in regulating cardiomyocyte function and demonstrated the functional benefit upon reversal of this modification [26, 27]. This is of clinical importance since it represents a potential treatment target to improve cardiac function in HCM. 


\section{Cell-to-cell mRNA/protein variability as pathomechanism in cardiomyopathy}

As familial cardiomyopathies represent an autosomal dominant genetic disorder, most patients are heterozygous for the mutation and carry one variant and one normal wild-type allele. In cardiomyopathy patients, the heart of a genotype-positive individual produces the variant protein in addition to the normal protein. As indicated above, the homeostasis of cellular proteins is tightly regulated by the PQC system, but it is also regulated at the mRNA level by non-sense mediated mRNA decay. Both systems are needed to suppress the accumulation of variant protein while keeping the normal protein at sufficient levels in cardiac muscle cells. It was recently shown that transcription of both alleles occurs independently and in a stochastic manner, where one cell favours one allele and the next cell favours the other allele [28]. This burst-like, stochastic on/off switching of allele transcription does not affect mRNA and protein levels in case of homozygous wild-type alleles. However, heterozygosity of alleles as present in genotype-positive individuals may introduce cell-to-cell variation with one cardiomyocyte expressing high levels of variant protein, while variant levels may be low in another cardiomyocyte [29]. Indeed, it has been shown that MYH7 gene variants cause a variable variant to wildtype ratio of mRNA expression in cardiomyocytes from the same heart $[28,30]$. The DOSIS consortium showed intercellular variation of cMyBP-C myofilament protein expression due to truncating $M Y B P C 3$ variants in the myocardium of HCM patients [31]. The functional consequences of the variable protein expression, which results in a mosaic pattern of cardiomyocytes with low and high variant/wildtype expression, remain to be determined. Loss of cMyBP-C causes severe dysfunction in mouse studies and engineered heart tissue [32, 33]. We propose that the intercellular variation of cMyBP-C protein levels causes inhomogeneous contraction and relaxation and underlies the formation of myofibrillar disarray, a currently unexplained disease characteristic of HCM. As ageing reduces the quality of PQC, an agedependent progression of the degree of allelic imbalance and cell-to-cell variation may contribute to cardiomyopathy development.

In conclusion, monogenetic cardiomyopathies have been intensely studied in the last three decades, and this has resulted in major progress in understanding what genes are involved. On the other hand, the striking heterogeneity, the highly variable age of onset, and the presence of gene variant carriers that never develop disease is as of yet largely unexplained. Given the profound repercussion for carriers, patients and family members we must improve our understanding of the individual's response to the presence of a pathogenic gene variant.
DOSIS aims to study unexplored mechanisms that will probably modify the pathogenic gene variant (Fig. 3). We have set up important initiatives and collaborations and have generated preliminary results showing that environmental and genetic modifiers are indeed important in our understanding. In the future we will step up our initiatives and projects and have identified an agenda, which contains-what we feel-important additional factors that when fully understood will guide clinicians in proper diagnosis, risk prediction, prognostication and, ultimately, cause-specific novel treatments.

Acknowledgements We thank Salva R. Yurista and Vasco Sequeira with the design of the Figures.

Funding CVON-DOSIS consortium 2014-40 Netherlands Heart Foundation. This work was supported by the Netherlands Heart Foundation [grant number 2015T041 to AFB and $\mathrm{MJ}]$.

Open Access This article is licensed under a Creative Commons Attribution 4.0 International License, which permits use, sharing, adaptation, distribution and reproduction in any medium or format, as long as you give appropriate credit to the original author(s) and the source, provide a link to the Creative Commons licence, and indicate if changes were made. The images or other third party material in this article are included in the article's Creative Commons licence, unless indicated otherwise in a credit line to the material. If material is not included in the article's Creative Commons licence and your intended use is not permitted by statutory regulation or exceeds the permitted use, you will need to obtain permission directly from the copyright holder. To view a copy of this licence, visit http://creativecommons.org/licenses/by/4.0/.

\section{References}

1. Walsh R, Thomson KL, Ware JS, et al. Reassessment of Mendelian gene pathogenicity using 7,855 cardiomyopathy cases and 60,706 reference samples. Genet Med. 2017;19:192-203.

2. Herman DS, Lam L, Taylor MR, et al. Truncations of titin causing dilated cardiomyopathy. N Engl J Med. 2012;366:619-28.

3. van der Zwaag PA, van Rijsingen IA, Asimaki A, et al. Phospholamban R14del mutation in patients diagnosed with dilated cardiomyopathy or arrhythmogenic right ventricular cardiomyopathy: evidence supporting the concept of arrhythmogenic cardiomyopathy. Eur J Heart Fail. 2012;14:1199-207.

4. van Spaendonck-Zwarts KY, van Rijsingen IA, van den Berg MP, et al. Genetic analysis in 418 index patients with idiopathic dilated cardiomyopathy: overview of 10 years' experience. EurJ Heart Fail. 2013;15:628-36.

5. van der Velden J, Stienen GJM. Cardiac disorders and pathophysiology of sarcomeric proteins. Physiol Rev. 2019;99:381-426.

6. Thomson KL, Ormondroyd E, Harper AR, et al. NIHR bioresource-rare diseases consortium. Analysis of 51 proposed hypertrophic cardiomyopathy genes from genome sequencing data in sarcomere negative cases has negligible diagnostic yield. Genet Med. 2019;21:1576-84.

7. van Lint FHM, Mook ORF, Alders M, Bikker H, Lekanne Dit Deprez RH, Christiaans I. Large next-generation sequenc- 
ing gene panels in genetic heart disease: yield of pathogenic variants and variants of unknown significance. Neth HeartJ. 2019;27:304-9.

8. Akinrinade O, Heliö T, Lekanne Deprez RH, et al. Relevance of titin missense and non-frameshifting insertions/ deletions variants in dilated cardiomyopathy. Sci Rep. 2019;9:4093.

9. Nijenkamp LLAM, Bollen IAE, van Velzen HG, et al. Sex differences at the time of myectomy in hypertrophic cardiomyopathy. Circ HeartFail. 2018;11:e4133.

10. van Driel B, Nijenkamp L, Huurman R, Michels M, van der Velden J. Sex differences in hypertrophic cardiomyopathy: new insights. Curr Opin Cardiol. 2019;34:254-9.

11. Huurman R, Schinkel AFL, van der Velde N, et al. Effect of body surface area and gender on wall thickness thresholds in hypertrophic cardiomyopathy. Neth Heart J. 2020;28:37-43.

12. Fumagalli C, Maurizi N, Day SM, et al. SHARE investigators. Association of obesity with adverse long-term outcomes in hypertrophic cardiomyopathy. JAMA Cardiol. 2019;6:1-8.

13. Robertson J, Schaufelberger M, Lindgren $M$, et al. Higher body mass index in adolescence predicts cardiomyopathy risk in midlife. Circulation. 2019;140:117-25.

14. Coats CJ, Heywood WE, Virasami A, et al. Proteomic analysis of the myocardium in hypertrophic obstructive cardiomyopathy. Circ Genom Precis Med. 2018;11:e1974.

15. Guclu A, Knaapen P, Harms HJ, et al. Disease stagedependent changes in cardiac contractile performance and oxygen utilization underlie reduced myocardial efficiency in human inherited hypertrophic cardiomyopathy. Circ Cardiovasc Imaging. 2017;10(5):e5604. https:// doi.org/ 10. 1161/CIRCIMAGING.116.005604.

16. Crilley JG, Boehm EA, Blair E, et al. Hypertrophic cardiomyopathy due to sarcomeric gene mutations is characterized byimpaired energy metabolismirrespective of the degree of hypertrophy. JAm Coll Cardiol. 2003;41:1776-82.

17. Ashrafian H, Redwood C, Blair E, Watkins H. Hypertrophic cardiomyopathy:a paradigm for myocardial energy depletion. Trends Genet. 2003;19:263-8.

18. Witjas-Paalberends ER, Guclu A, et al. Gene-specific increase in the energetic cost of contraction in hypertrophic cardiomyopathy caused by thick filament mutations. Cardiovasc Res. 2014;103:248-57.

19. Verdonschot JAJ, Hazebroek MR, Derks KWJ, et al. Titin cardiomyopathyleads to altered mitochondrial energetics, increased fibrosis and long-term life-threatening arrhythmias. Eur Heart J. 2018;39:864-73.

20. van der Velden J, Tocchetti CG, Varricchi G, et al. Metabolic changes in hypertrophic cardiomyopathies. Scientific update from the working group of myocardial function of the ESC. Cardiovasc Res. 2018;114:1273-80.

21. van Driel BO, Rossum AC, Michels M, Huurman R, van der Velden J. Extra energy for hearts with a genetic defect: ENERGY trial. Neth Heart J.2019;27:200-5.

22. Jansen M, Baas AF, van Spaendonck-Zwarts KY, et al. Mortality risk associated with truncating founder mutations in titin. Circ Genom Precis Med. 2019;12:e2436.

23. Te Rijdt WP, van der Klooster ZJ, HoorntjeET, et al. Phospholamban immunostaining is a highly sensitive and specific method for diagnosing phospholamban p.Arg14del cardiomyopathy. Cardiovasc Pathol. 2017;30:23-6.

24. Dorsch LM, Schuldt M, Knežević D, et al. Untying the knot: protein quality control in inherited cardiomyopathies. Pflugers Arch. 2019;471:795-806.

25. Dorsch LM, Schuldt M, dos Remedios CG, et al. Protein quality control activation and microtubule remodeling in hypertrophic cardiomyopathy. Cells. 2019;18:8.

26. Robison P, Caporizzo MA, Ahmadzadeh H, et al. Detyrosinated microtubules buckle and bear load in contracting cardiomyocytes. Science. 2016;352(6284):aaf659.

27. Chen CY, Caporizzo MA, Bedi K, et al. Suppression of detyrosinated microtubules improves cardiomyocyte function in human heartfailure. Nat Med. 2018;24:1225-33.

28. Montag J, Kowalski K, Makul M, et al. Burst-like transcription of mutant and wildtype MYH7-alleles as possible origin of cell-to-cell contractile imbalance in hypertrophic cardiomyopathy. Front Physiol. 2018;9:359.

29. Kraft T, Montag J, Radocaj A, Brenner B. Hypertrophic cardiomyopathy: cell-to-cell imbalance in gene expression and contraction force as trigger for disease phenotype development. Circ Res. 2016;119:992-5.

30. Brenner B, Seebohm B, Tripathi S, Montag J, Kraft T. Familial hypertrophic cardiomyopathy: functional variance among individual cardiomyocytes as a trigger of FHC-phenotype development. Front Physiol. 2014;5:392.

31. Parbhudayal RY, Garra A, Götte MJW, et al. Variable cardiac myosin binding protein-C expression in the myofilaments due to MYBPC3 mutations in hypertrophic cardiomyopathy. J Mol Cell Cardiol. 2018;123:59-63.

32. McConnell BK, Jones KA, Fatkin D, et al. Dilated cardiomyopathy in homozygous myosin-binding protein-C mutant mice. J Clin Invest. 1999;104:1235-44.

33. Wijnker PJM, Friedrich FW, Dutsch A, et al. Comparison of the effects of a truncating and a missense MYBPC3 mutations on contractile parameters of engineered heart tissue. J Mol Cell Cardiol. 2016;97:82-92.

34. Sequeira V, Nijenkamp LL, Regan JA, van der Velden J. The physiological role of cardiac cytoskeleton and its alterations in heartfailure. Biochim Biophys Acta. 2014;1838:700-22. 